\title{
La disciplina delle biotecnologie tra scelte di politica comune e sviluppo territoriale
}

\author{
Matteo Benozzo ******
}

Sommario: 1. Introduzione. - 2. Il "bisogno di salubrità" nelle economie occidentali: i modelli di approccio a livello mondiale. - 3. Le differenti scelte di regolazione delle biotecnologie. - 4. La disciplina di filiera dell'Unione Europea. - 5. Una componente della scelta di fondo nell'Unione Europea tra politica comune e sviluppo territoriale. 6. Conclusioni.

\section{Introduzione}

In materia di biotecnologie alimentari «l'obbligo di autorizzazione e di vigilanza sussiste a prescindere dalla proporzione di materiale geneticamente modificato contenuta nel prodotto di cui trattasi» e ciò «indipendente dal carattere intenzionale o accidentale dell'introduzione della sostanza di cui trattasi».

E' quanto ha sentenziato la Corte di giustizia in una recentissima pronuncia $^{1}$, il cui contenuto rappresenta riprova dell'approccio delle istituzioni europee e nazionali alla questione delle biotecnologie utilizzate in campo alimentare. Secondo tale approccio, gli organismi geneticamente modificati (ogm) sarebbero prodotti a salubrità incerta e, per tale motivo, da sottoporre ad una gestione vincolata di governo dell'intera filiera,

\footnotetext{
* Università degli studi di Macerata

** L'articolo riproduce l'elaborato destinato agli atti del II Convegno nazionale promosso dall'Associazione Internazionale "per il Sostegno e la Valorizzazione delle Attività Cistercensi nel Mondo", in collaborazione con la Fondazione "Studi e Ricerche su Alimentazione e Salute", tenuto presso il Monastero Cistercense di Martano (Lecce) nei giorni 1-2 ottobre 2011, sul tema "Alimentazione, Salute e Ambiente.

${ }^{* * *}$ L'intervento ha per oggetto un argomento che rientra nella ricerca Prin 2008 «Agricoltura e crisi alimentari. Strategie, regole e tecniche» (prot. 2008M2C4NR), coordinata dal prof. F. Adornato, e in particolare, nelle attività dell'unità dell'Università di Macerata sotto il titolo «Regole giuridiche e sostenibilità alimentare nelle produzioni agroenergetiche, biotecnologiche e di qualità» di cui è responsabile il prof. F. Adornato

1 Corte di giustizia 6 settembre 2011, causa C-442/09, Bablok v. Monsanto, in http://curia.europa.eu, i cui passaggi riportati nel testo riproducono quanto, rispettivamente, $\mathrm{i}$ punti 103 e 89 della sentenza, già commentata nella portata e nelle conseguenze da Masini $2011,546$.
} 
dall'ideazione, alla fase sperimentale e fino al consumo finale, cui il regime autorizzatorio previsto nelle singole regole in materia sarebbe funzionale alla creazione di un sistema generalizzato di controllo e regolamentazione di tutte le attività che ineriscono o, comunque, sono interessate dalla presenza, anche non più attuale, di ogm.

Siffatta gestione vincolata risponde ad una scelta ben precisa, conseguenza del crescere di un "bisogno di salubrità", prima ancora che nell'Unione Europea, nel mercato mondiale dei prodotti alimentari in cui la purezza e l'igiene dell'alimento stanno diventando sempre più caratteristiche determinanti ed irrinunciabili nella formazione del consenso negli acquisti.

\section{Il "bisogno di salubrità" nelle economie occidentali: i modelli di approccio a livello mondiale}

L'introduzione delle biotecnologie avanzate nel mercato alimentare e il crescere dell'attenzione dei consumatori sulla food safety (Borghi 2003, 449) nelle economie del nord del mondo, hanno visto nascere diversi approcci giuridici alla materia alimentare, quale conseguenza della diversa percezione del "bisogno di salubrità".

Nei singoli Stati, invero, tale "bisogno di salubrità" non ha la medesima incidenza e le medesime manifestazioni, ma si presenta con connotazioni diverse secondo la particolare sensibilità dei legislatori statali chiamati a farsene interpreti, dove la cultura, la tradizione, le origini e le scelte alla base del singolo sistema, ne influenzano l'espressione e il peso.

Allo stato, dei vari modelli di food safety, due in particolare si stanno imponendo maggiormente a livello mondiale: il modello comunitario e il modello statunitense.

Il primo limita la libera circolazione degli alimenti e dei mangimi nei casi di insicurezza alimentare conclamata (Costato 2010, 35; Costato, Borghi e Rizzoli 2011) e, amplificando la portata del "bisogno di salubrità" con il principio di precauzione ${ }^{2}$, interviene sul mercato anche in ipotesi di "dubbio non smentito" di salubrità, dove l'incertezza impedisce la libera circolazione delle merci (Bruno 2000, 569; Gradoni 2003, 204; Gragnani 2003, 9). Nei Paesi che adottano tale modello, la lettura precauzionale del rischio consente il commercio alimentare solo a prodotti a "salubrità piena" (Germanò e Rook Basile 2005, 223), impegnando il singolo produttore, l'importatore, il distributore e anche le stesse autorità pubbliche ad uno sforzo di valutazione e

\footnotetext{
${ }^{2}$ In generale, sul principio di precauzione la bibliografia è oramai molto ampia, si v. per tutti, da ultimo, Borghi 2011, 53.
} 
gestione del rischio prima dell'immissione del prodotto sul mercato (Adornato 2005, p. 761).

Un siffatto modello, infatti, si fonda sul divieto di commercializzazione degli alimenti "a rischio" e la valutazione del grado di rischiosità e la relativa gestione sono attività che devono necessariamente compiersi prima dell'immissione del prodotto sul mercato. Il singolo produttore (o importatore) è sempre tenuto a dar prova della salubrità del prodotto alle pubbliche autorità prima della sua commercializzazione, autorità che, valutato il rischio, decidono se esporre o meno la collettività ad esso, consentendo o meno la libera circolazione dell'alimento, sottoponendola a condizioni ovvero vietandone il commercio.

Il secondo modello che esprime un peculiare approccio ai rischi alimentari è quello statunitense dove il "bisogno di salubrità" si è venuto imponendo al sistema giuridico, generando regole limitative della libera circolazione delle merci. Sennonché, rispetto al modello comunitario, quello statunitense si è venuto "contaminando" da interpretazioni liberali e liberiste, portandolo a concepire limitazioni alla libera circolazione delle merci solo in caso di rischio alimentare evidente e quando il singolo produttore non ritiene di essere in grado di gestirne autonomamente le conseguenze (Benozzo 2002, 213). Una scelta interpretativa, questa, rilevabile nella disciplina di regolamentazione dell'intera filiera alimentare americana (Teel 2000, 649; Kysar 2004, 525), comprensiva anche della produzione, circolazione e utilizzazione dei mangimi (Benozzo 2004a, 63).

Se al pari del primo anche il modello statunitense riconosce un divieto generale di produrre, importare, distribuire o commercializzare alimenti insalubri nel territorio nazionale, una serie di eccezioni a tale divieto e la possibilità che l'applicazione di tali eccezioni sia rimessa alle decisioni del singolo produttore, i momenti di valutazione e gestione dei rischi vengono a separarsi in due fasi distinte, rispettivamente, nella pre e nella post-market fase. Prima della commercializzazione di un nuovo prodotto, invero, se formalmente, per il divieto generale indicato, ogni produttore è sempre obbligato ad avviare una pre-market procedure di valutazione dell'eventuale rischio e di sua gestione preventiva, in sostanza tale procedura è sempre eventuale e successiva; ossia, il rischio incerto non è gestito in via preventiva, ma solo ove il singolo produttore lo ritiene necessario, assumendosi questi il rischio di esporre la collettività all'incertezza, al pericolo non certo di un evento dannoso. Se poi tale pericolo si concretizza effettivamente nell'evento, solo allora si procede con la risk management. Ossia, questo peculiare funzionamento ha l'effetto di assimilare il settore alimentare agli altri settori imprenditoriali che sono creati sul modello statunitense, in cui la gestione del rischio conseguente all'eventuale dannosità di un prodotto è rimessa 
totalmente al mercato e l'intervento pubblico, sebbene destinato ad anticipare, come nel diritto europeo (Bruno 2003, 238; Lattanzi 2004, 237), nocumenti da prodotti "a rischio", è di fatto quasi sempre successivo alla commercializzazione del prodotto stesso (Germanò 2002, 323).

I due modelli indicati, quindi, si presentano profondamente diversi. Mentre il modello europeo è venuto costruendo un sistema di food safety che limita la libera circolazione degli alimenti e dei mangimi, non solo nei casi di insicurezza alimentare certa, ma anche nelle ipotesi di "dubbio non smentito" di salubrità, dove l'incertezza impedisce la piena libertà del mercato a tutela dei consumatori (approccio c.d. "precauzionale" o anche market-oriented), il modello statunitense ha riconosciuto tale "bisogno di salubrità" filtrandolo attraverso una interpretazione restrittiva, che consente limitazioni alla circolazione delle merci solo nei casi di rischio alimentare conclamato senza tutele preventive per i consumatori (approccio c.d. "preventivo" o anche product-oriented) (Jannarelli 2004, 5). Ossia, mentre nel primo sistema l'approccio precauzionale amplifica la portata del "bisogno di salubrità" fino a farvi ricomprendere letture conservative e di tutela con interventi diretti in situazioni di incertezza sulla salubrità di un prodotto, nel secondo, la mancanza di certezza non limita mai la "libera circolazione delle merci".

\section{Le differenti scelte di regolazione delle biotecnologie}

I differenti modelli indicati si sostanziano per le biotecnologie in discipline profondamente diverse.

Negli Stati Uniti, il modello "preventivo" o product-oriented applicato alle biotecnologie ha portato ad una lettura giuridica delle vicende del medesimo oggetto articolata nel duplice approccio della dicotomia "product of nature human made invention" e del principio della "sostanziale equivalenza".

Con la dicotomia "product of nature - human made invention", il prodotto biotech viene riconosciuto come una vera e propria invenzione industriale suscettibile di brevetto che assicura al suo titolare il massimo della tutela giuridica attribuibile al risultato di un'attività inventiva. Con il principio della "sostanziale equivalenza", invece, il medesimo prodotto biotech perde la sua specificità per essere assimilato ai prodotti convenzionali consentendone l'allocazione sul mercato nel rispetto delle medesime regole (Benozzo 2004b, 129 e Benozzo e Bruno 2006, 709). Ossia, considerando le tecniche del dna ricombinante come un mero sviluppo delle tradizionali tecniche di manipolazione genetica (Franken 2000, 117), i prodotti delle prime sono considerati a tutti gli effetti identici o simili a quelli realizzati attraverso le seconde, in quanto entrambi governati da medesime leggi fisiche e biologiche 
(Francer 2000, 267).

Nell'Unione Europea, invece, il modello "precauzionale" o marketoriented di food safety applicato alle biotecnologie si è concretizzato nel principio della "sostanziale diversità". Tale principio, pur non impedendo la messa in commercio degli alimenti geneticamente modificati (gm), ponendo l'accento sull'origine "non naturale" del prodotto (ossia sul procedimento biotecnologico che ne ha dato la luce), ne consente la commercializzazione solo previa autorizzazione a valle di un procedimento che garantisca l'assenza di rischi.

Dopo l'autorizzazione, poi, la circolazione del prodotto non è libera ma sottoposta a specifiche regole (come etichettatura, rispetto di determinate distanze nella coltivazione ed altro), regole che rispetto all'omologo convenzionale, evidenziano l'assoluta diversità dell'alimento biotech che si fa unicità nella disciplina sui brevetti, rendendo suscettibile il titolare della "creazione" della protezione del proprio "ritrovato".

La "sostanziale diversità", diretta emanazione del principio di precauzione, è in materia alla base dell'azione della Comunità che già dalla seconda metà degli anni ottanta, è venuta occupandosi di biotecnologie allo scopo dichiarato di assicurare un'elevata sicurezza dell'ambiente e della salute pubblica. Dall'inizio degli anni Novanta, invero, sono stati emanati una serie di provvedimenti strutturali che, anche in forza del regolamento 28 gennaio 2002 , n. 178/2002 sulla sicurezza alimentare ${ }^{3}$, garantiscono oggi un quadro normativo completo volto a disciplinare, in una visione di filiera del prodotto biotecnologico, i materiali gm dall'ideazione al loro utilizzo finale (Benozzo 2011, 149).

\section{La disciplina di filiera dell'Unione Europea}

Le biotecnologie alimentari rientrano sia nella materia agricoltura, sia nella materia alimentazione per la determinazione delle competenze legislative. Mente la prima materia è concorrente dell'Unione Europea per la fase di coltivazione ed esclusiva per il funzionamento del mercato interno (artt. 3 e 4 del Trattato sul funzionamento dell'Unione Europea - TFUE), nella materia alimentazione in senso ampio e, quindi, nella sua duplice componente della food security (agricoltura: Albisinni 2005) e della food safety a tutela della sanità pubblica e dei consumatori (artt. 168 e 169 del TFUE: Germanò 2007,

\footnotetext{
${ }^{3}$ In generale, sulla disciplina di cui al regolamento indicato, la bibliografia è ormai vastissima; ci sia consentito limitare il richiamo, anche per riferimenti bibliografici, ai soli contributi pubblicati in Costato, Rook Basile e Germanò 2011.
} 
24), la Comunità ricopre un ruolo centrale nella formazione del relativo diritto, lasciando allo Stato limitatissimi margini di manovra (Benozzo 2008, 114).

Il quadro normativo che l'Europa è venuta dipingendo per le biotecnologie dalla seconda metà degli anni ottanta, come detto, ha una visione di filiera del relativo prodotto. Ogni fase di gestione del prodotto biotech è disciplinata con una serie di provvedimenti orizzontali riguardanti l'intera gamma dei beni realizzabili, dai materiali microbiologici gm e dalla loro ideazione, fino all'utilizzo finale e al consumo del prodotto generato come alimento.

Così, partendo dalla direttiva 23-4-1990, n. 90/220, sull'emissione deliberata nell'ambiente e la messa in commercio degli ogm, la disciplina comunitaria si è venuta arricchendo delle direttive 6-7-1998, n. 98/44, sulla brevettazione del materiale genetico e 12-3-2001, n. 2001/18, che abroga e sostituisce la precedente 90/220 (Sirsi 2011, 269). Inoltre, nel medesimo quadro normativo, sono stati emanati il regolamento 27-1-1997, n. 258/97, sull'immissione in commercio dei "novel foods", ogm o da essi derivati, ed i regolamenti sulla etichettatura dei prodotti immessi in commercio 26-5-1998, n. 1139/98, 10-1-2000, n. 49/2000 e 10-1-2000, n. 50/2000 (Benozzo e Bruno 2003). Infine, l'intera disciplina sugli alimenti gm è stata riformata con l'introduzione dei due regolamenti CE del Parlamento europeo e del Consiglio 22-9-2003, n. 1829/2003 e n. 1830/2003 (meglio conosciuti come regolamenti "food and feed"), che hanno abrogato per gli ogm quello sui novel foods e le disposizioni specifiche sull'etichettatura prima in vigore (Valletta 2005) ${ }^{4}$. Da ultimo, poi, il quadro è stato completato con il regolamento $\mathrm{CE}$ del Parlamento europeo e del Consiglio 15-7-2003, n. 1946/2003 di attuazione del protocollo di Cartagena sulla biosicurezza, convenzione sulla diversità biologica che mira a stabilire un sistema comune di notifiche e di scambio di informazioni sui movimenti transfrontalieri di ogm verso paesi terzi, onde garantire che i movimenti atti ad avere effetti negativi sull'uso sostenibile della diversità biologica e sulla salute umana avvengano rispettando l'ambiente e la salute umana (Borghi 2001, 365 e Righini 2001, 654).

A livello nazionale, le procedure di recepimento del quadro normativo comunitario hanno portato all'emanazione di provvedimenti legislativi il cui contenuto ricalca perfettamente gli atti comunitari, aggiungendo ad essi

\footnotetext{
${ }^{4} \mathrm{Nel}$ completamento del quadro giuridico di filiera, è ricompresa anche la direttiva 23 aprile 1990, n. 90/220 sull'utilizzo confinato dei microrganismi geneticamente modificati, modificata dalla direttiva 26 luglio 1998, n. 95/81 e oggi oggetto di "rifusione" in una nuova direttiva che abroga e sostituisce la prima: la direttiva del Parlamento europeo e del Consiglio 6 maggio 2009, n. 2009/41.
} 
unicamente le disposizioni necessarie a renderli operativi e clausole sanzionatorie specifiche. Così: la direttiva n. 90/220 è stata attuata con il d.lg. 3-3-1993, n. 92; la direttiva n. 98/44 ha visto adozione con il d.1. 10-1-2006, n. 3 (convertito con modifiche il 22 febbraio 2006 nella legge n. 78); e la direttiva n. 2001/18 è entrata in vigore con il d.lg. 8-7-2003, n. 224. I due regolamenti nn. 1829/2003 e 1830/2003, invece, hanno trovato copertura sanzionatoria con il d.lg. 21-3-2005, n. 70, il cui contenuto, da ultimo, stabilisce sanzioni amministrative pecuniarie e sanzioni penali (alternativamente l'arresto o l'ammenda) collegate alle varie condotte illegali, non distinguendo, nella irrogazione delle sanzioni per violazione delle norme sull'etichettatura, tra grandi gruppi industriali e medi e piccoli operatori alimentari e agricoli, con una ingente sanzione amministrativa pecuniaria in caso di commercializzazione, anche involontaria, di alimenti e mangimi contenenti ogm.

Il reticolo di norme e regole che ne derivano, quindi, è tale da comprimere notevolmente la gestione e l'utilizzo dei materiali biotecnologici in assenza di previ controlli; basti come esempio accennare al fatto che le fasi di sperimentazione, di emissione nell'ambiente a scopo di ricerca e sviluppo, di emissione a scopo di coltivazione e di immissione sul mercato alimentare o mangimistico per il consumo, sono tutte caratterizzate da un autonomo e specifico procedimento autorizzatorio volto al rilascio di una autorizzazione ad hoc, la cui mancanza impedisce l'utilizzo legittimo del materiale gm. Di più: siffatto procedimento non è meramente di verifica documentaleburocratica, ma per ogni fase il singolo ogm è sottoposto ad una specifica analisi di rischio, composta da un momento di valutazione del rischio, di risk assessment, di competenza normalmente, per l'ingresso nel mercato alimentare e mangimistico, dell'EFSA (ossia, dell'Autorità per la sicurezza alimentare che ha sede a Parma: Benozzo 2003, 303), e da un momento di sua gestione, di risk management, nella disponibilità solita dell'organo politicoamministrativo europeo, la Commissione. Anche ottenuta l'autorizzazione (sempre temporanea), poi, il materiale gm può essere sempre interessato da: a) condizioni particolari e successive di utilizzo; b) sospensioni o interruzioni nell'emissione od immissione; $c$ ) assunzione di una qualsiasi misura "adeguata" a contrastare la situazione riscontrata in seguito, potendosi giungere finanche alla revoca del provvedimento autorizzativo.

Oltre l'autorizzazione, inoltre, la circolazione del materiale gm richiede sempre l'utilizzo di apposita etichetta. Il produttore (od importatore), invero, deve palesare al consumatore la natura biotecnologica del prodotto e mettere a

\footnotetext{
${ }^{5}$ Artt. 53 e 54 del regolamento n. 178/2002, richiamati dal regolamento in esame, su cui v. Albisinni 2003, 440.
} 
disposizione del mercato quell'informazione ritenuta material ("essenziale", "determinante") sull'origine "non naturale", non recuperabile in altro modo dal consumatore (Benozzo 2011, 149). A tale riguardo, specifici requisiti di etichettatura e tracciabilità sono previsti per gli ogm e gli alimenti contenenti o costituiti o prodotti da ogm (presenti volontariamente o con coscienza ovvero in proporzioni superiori allo $0,9 \%$ ), con obbligo di comunicazione, se del caso, anche della diversa composizione, natura, effetti nutrizionali od uso ed implicazioni per la salute rispetto all'omologo convenzionale (Bruno 2005, 159; Id. 2009, 47).

\section{Una componente della scelta dell'Unione Europea tra politica comune e sviluppo territoriale}

L'approccio precauzionale (o market-oriented) della Comunità nel settore alimentare, ha portato nelle biotecnologie, come visto, alla strutturazione di un sistema ad applicazione orizzontale che ruota intorno alla sicurezza del consumatore e alla sua integrale tutela, con una disciplina fondata sulla "sostanziale diversità" dei prodotti biotech rispetto agli omologhi convenzionali e regole proprie sulla commercializzazione ed etichettatura che si sovrappongono alla disciplina ordinaria. L'intero sistema è strutturato nella convinzione che dinanzi al dubbio di salubrità di siffatti prodotti, una valutazione e gestione dei rischi preventiva ed una corretta comunicazione al pubblico sia l'approccio più "naturale".

In questi termini, la posizione della Corte di giustizia su «l'obbligo di autorizzazione e di vigilanza [che] sussiste[rebbe] a prescindere dalla proporzione di materiale geneticamente modificato contenuta nel prodotto di cui trattasi》 e ciò «indipendente dal carattere intenzionale o accidentale dell'introduzione della sostanza», trova facile giustificazione, ma richiede un'ulteriore valutazione di una componente di fondo della posizione di tutela assunta, che impedisce un qualsiasi uso sul mercato di prodotti di dubbia origine bioingegneristica, finanche a ricomprendervi quelli contaminanti senza la consapevolezza del produttore, senza la sua partecipazione e anche se per ogm presenti in proporzioni leggermente superiori al limite dello 0,9\%. Inoltre, ad autorizzazione ottenuta, ossia sottoposto a controllo preventivo di identificazione dell'eventuale natura o contaminazione gm, i relativi prodotti "contaminati" possono essere utilizzati e circolare solo - e qui forse, in modo eccessivo - rispettando ferree regole di comunicazione.

I prodotti gm autorizzati, invero, non entrano sul mercato alle medesime regole dei loro omologhi convenzionali. Essi devono sottostare a specifiche norme sull'etichettatura, le quali sembrerebbero fondate, più che sul 
perseguimento - giustamente e condivisibile - della salubrità e della tutela anche precauzionale della salute pubblica e dell'ambiente (fine precipuo delle autorizzazioni), sulla consapevolezza del ruolo della comunicazione. Interesse preminente qui, è il fronteggiare i pericoli della distorsione della comunicazione soprattutto quando ad essere a rischio sono l'indipendenza e la libertà del consumatore, con conseguenze collegate alla possibile evoluzione della scienza relativa a prodotti della bioingegneria (Benozzo 2011, 149).

In sostanza, quindi, l'indicazione in etichetta dell'origine bioingegneristica dirotta il prodotto autorizzato e a salubrità preventivamente accertata verso un mercato parallelo a bassa redditività. Tale mercato, poi, è ulteriormente svantaggiato rispetto a quello ordinario dei prodotti convenzionali dalla politica informativa che la stessa Comunità negli ultimi anni ha seguito, amplificando quella percezione negativa verso gli ogm diffusa presso i propri consumatori.

Posta in questi termini, quindi, la giustificazione di una siffatta disciplina di filiera con il solo richiamo dell'approccio "precauzionale" europeo non appare del tutto completa o, addirittura, del tutto soddisfacente, necessitando l'atteggiamento comunitario verso gli ogm, specie per le regole proprie dell'ultima fase di circolazione sul mercato, di indagare una ulteriore possibile motivazione.

Ebbene, l'adattamento di un sistema giuridico a prodotti a "salubrità incerta", come di fatto sono gli ogm, non è unicamente effetto dell'approccio precauzionale o meno del singolo ordinamento e della filosofia alla base e della cultura propria della società (Germanò 2002, 323), ma anche conseguenza diretta di una scelta fondamentalmente di politica economica.

Ed invero, sostenere o meno le biotecnologie alimentari rappresenta una decisione che, nella mancanza di certezza di medio lungo termine, si concretizza in una sostanziale "scommessa" dove il consentire o meno l'adattamento del sistema economico allo sfruttamento di un siffatto prodotto, espone il sistema stesso ad un rischio per la salute pubblica e la stabilità economica delle imprese coinvolte, di notevole portata che solo il potere politico può assumersene la responsabilità, giustificandola, ove valida ed efficace, per lo sviluppo economico del relativo paese (Benozzo 2011, 149).

Nell'Unione Europea, la scelta di approccio sugli ogm, oltre al terreno fertile di una lettura filosofico-culturale tipica delle nostre tradizioni, sembrerebbe essere quella di non "scommettere" sulle biotecnologie anche per la mancanza di valore aggiunto per il suo sistema agroalimentare. Come è noto, invero, siffatto sistema è fortemente interconnesso con i contesti territoriali e culturali delle varie regioni dei Paesi membri, dove - salvo alcune aree - la produzione di massa e la messa in coltura di ogm, caratterizzati sostanzialmente sulla spersonalizzazione e sull'appiattimento del gusto, non 
sembrano rappresentare un vantaggio per gli operatori agricoli ed alimentari europei. Ecco quindi la scelta di sposare la "qualità" e la "identità" dei prodotti per lo sviluppo del comparto (Adornato 2009, 6) e la "avversione" alle biotecnologie come imposte sul mercato dagli Usa (Benozzo e Bruno 2006, 709).

\section{Conclusioni}

Alcune considerazioni conclusive.

Nel campo delle biotecnologie, il differente bisogno di salubrità dei singoli ordinamenti e la conseguente più o meno evidente sensibilità verso la sicurezza alimentare e le relative scelte che ne possono derivare in termini di livello di tutela, rappresentano la componente principale delle possibili motivazioni di approccio alla materia. Il modello comunitario, in questi termini, è venuto modellando una disciplina stringente e limitativa delle libere circolazione ed utilizzo degli ogm, caratterizzando le attività imprenditoriali in materia da una notevole eteroderminazione.

Le scelte autorizzative preventive sugli ogm, infatti, rispondono alla piena e diretta applicazione del principio di precauzione, nonché al principio di responsabilità delle imprese e degli individui alle esigenze della società attuale e delle generazioni future, che porta a preferire l'astensione e la limitazione dell'operare ove il muoversi nel territorio dell'incertezza, può esporre la collettività a rischi mai adeguatamente e completamente risarcibili.

$\mathrm{Si}$ giunge poi alla valutazione delle regole sull'etichettatura, dove l'etichetta non è più solo il luogo dove avviene il dialogo tra produttori e consumatori e dove il "produttore riporta le informazioni che ritiene opportune e quelle che la legge gli impone» (Germanò e Rook Basile 2002, 138), ma viene a rappresentare un elemento imprescindibile di una scelta consapevole che il legislatore comunitario vuole tutelare; luogo, questo, da cui il consumatore «ricava tutto ciò che gli serve per "sapere" che cosa sia quel prodotto, di quali qualità sia provvisto, da quali pregi o manchevolezze sia caratterizzato» (Idem).

Rispetto agli ogm, quindi, l'informazione al cittadino dell'origine transgenica dell'alimento che si accinge ad ingerire, risponde a tali esigenze. Nella valutazione complessiva del sistema in cui viene a funzionare la relativa regola, però, l'etichetta per gli ogm è anche qualcosa in più: un modo per trasferire dalla collettività ai produttori il rischio insito nell'incertezza dovuta all'evoluzione della scienza, nonché un espediente per limitare la diffusione di prodotti non "convenienti" per l'economia europea, sollecitando un deperimento della relativa domanda. 


\section{Bibliografia}

Albisinni F. (2003), «Commento agli articolo 53 e 54», in Idaic - Istituto di Diritto Agrario Internazionale e Comparato (a cura di), La sicurezza alimentare nell'Unione Europea: commento al Reg. n. 178/02/CE, in Nuove leggi civili commentate, n. 1-2, p. 440-443

Albisinni F. (2005), Profili di diritto europeo per l'impresa agricola. Il regime di aiuto unico e le attività dei privati, Viterbo.

Adornato F. (2009), «Le "declinazioni della qualità": una nota introduttiva», in Rivista di Diritto Alimentare, n. 3, p. 6-20

Adornato F. (2005), «La sicurezza alimentare tra mercato unico e diritto comune europeo», in Rivista di Diritto Agrario, n. 4, p. 761-769

Benozzo M. (2002), «La disciplina statunitense delle biotecnologie in agricoltura», in Germanò (a cura di), La disciplina giuridica dell'agricoltura biotecnologia, Milano, 2002, 213-322.

Benozzo M. (2003), «Commento agli artt. 22 e 23», in Idaic - Istituto di Diritto Agrario Internazionale e Comparato (a cura di), ult.op.cit., n. 1-2, p. 290-306

Benozzo M. (2004a), «Mangimi, ormoni e antibiotici: tecniche statunitensi di allevamento», in Agricoltura Istituzioni Mercati, n. 3, p. 63-92.

Benozzo M. (2004b) «Stati Uniti d'America e biotecnologie in agricoltura: un nuovo approccio al problema», in Agricoltura Istituzioni Mercati, n. 1, p. $129-146$

Benozzo M. (2008), «Alimentazione: una materia trasversale», in Issirfa - Cnr (a cura di), Regioni e attività produttive. Rapporto sulla legislazione e sulla spesa: 2005-2007. Il punto sulle materie, Milano, p. 107-125.

Benozzo M. (2011), «Alimenti geneticamente modificati», in Costato, Rook Basile e Germanò (diretto da), Trattato di diritto agrario. Volume terzo: il diritto agroalimentare, Torino, 2011, p. 149-183.

Benozzo M. e Bruno F. (2003), Legislazione ambientale, Milano

Benozzo M. e Bruno F. (2006), La disciplina delle biotecnologie tra diritto europeo e diritto statunitense, in Diritto e giurisprudenza agraria, alimentare e dell'ambiente, n. 12, p. 709-718

Borghi P. (2001), «Biotecnologie, tutela dell'ambiente e tutela del consumatore nel quadro normativo internazionale $e$ nel diritto comunitario», in Rivista di Diritto Agrario, n. 3, I, p. 365-409

Borghi P. (2003), «Sicurezza alimentare e commercio internazionale», in Rook Basile, Massart e Germanò (a cura di), Prodotti agricoli e sicurezza alimentare. Volume I, Milano, 449-470. 
Borghi P. (2011), «Il rischio alimentare e il principio di precauzione», in Costato, Rook Basile e Germanò (diretto da), Trattato di diritto agrario. Volume terzo: il diritto agroalimentare, cit., p. 53-72

Bruno F. (2000), «Il principio di precauzione tra diritto dell'Unione Europea e WTO», in Diritto e giurisprudenza agraria, alimentare e dell'ambiente, $\mathrm{n}$. 10, p. $569-577$

Bruno F. (2003), «Commento all'art. 14», in Idaic - Istituto di Diritto Agrario Internazionale e Comparato (a cura di), La sicurezza alimentare nell'Unione Europea: commento al Reg. n. 178/02/CE, cit., p. 238-246

Bruno F. (2005), Biotecnologie e comunicazione: le etichette dei prodotti geneticamente modificati, in Germanò e Rook Basile (a cura di), Il diritto alimentare tra comunicazione e sicurezza dei prodotti, Torino, p. 159-184.

Bruno F. (2009), «Le etichette degli Ogm», in Agricoltura Istituzioni Mercati, n. $1-2$, p. $47-54$

Costato L. (2010), «Protezione del consumatore tra strumenti contrattuali e norme di carattere pubblicistico: il caso del diritto alimentare», in Rivista di diritto agrario, n. 1, I, p. 35-46

Costato L., Borghi P. e Rizzoli S. (2011), Compendio di diritto alimentare, Padova, 2011

Costato L., Rook Basile E. e Germanò A. (2011) (diretto da), Trattato di diritto agrario. Volume terzo: il diritto agroalimentare, Torino

Francer J.K. (2000), «Frankenstein Foods or Flavor Savers?: Regulating Agricultural Biotechnology in the United States and European Union», in Virginia Journal of Social Policy and the Law, n. 7, 257-314.

Franken M., «Fear of Frankenfoods: A Better Labeling Standard for Genetically Modified Foods», in Minnesota Intellectual Property Review, 2000, n. 1, 117-132.

Germanò A. (2002), «Gli aspetti giuridici dell'agricoltura biotecnologica», in Germanò (a cura di), La disciplina giuridica dell'agricoltura biotecnologia, Milano, p. 323-360.

Germanò A. (2007), Corso di diritto agroalimentare, Padova.

Germanò A. e Rook Basile E. (2002), La disciplina comunitaria e internazionale del mercato dei prodotti agricoli, Torino.

Germanò A. e Rook Basile E. (2005), «La sicurezza dei prodotti alimentari», in Germanò e Rook Basile (a cura di), Il diritto alimentare tra comunicazione e sicurezza dei prodotti, cit., p. 223-316.

Gradoni L. (2003), «Commento all'art. 7», in Idaic - Istituto di Diritto Agrario Internazionale e Comparato (a cura di), ult. op. cit., p. 204-217.

Gragnani A. (2003), «Il principio di precauzione come modello di tutela dell'ambiente, dell'uomo e delle generazione future», in Rivista di diritto civile, n. 1, II, p. 9 - 45 
Kysar D.A. (2004), «Preferences for processes: the process/product distinction and the regulation of consumer choice», in Harvard Law Review, p. 525-642

Jannarelli A. (2004), «La qualità dei prodotti agricoli: considerazioni introduttive ad un approccio sistematico», in Diritto e giurisprudenza agraria, alimentare e dell'ambiente, n. 1, p. 5 - 13.

Lattanzi P. (2004), «Il sistema di allarme rapido nella sicurezza alimentare», in Agricoltura Istituzioni Mercati, n. 3, p. 237.262;

Masini S. (2011), «Sul dialogo tra scienza e giudici in materia di api e OGM», in Diritto e giurisprudenza agraria, alimentare e dell'ambiente, n. 9, pp. 546-548.

Righini E. (2001), Il protocollo di Cartagena sulla biosicurezza e gli accordi sul commercio internazionale, in Rivista di diritto internazionale, n. 3, p. 654-685.

Sirsi E. (2011), «L'impiego in agricoltura di organismi geneticamente modificati e la coesistenza con le coltivazioni non geneticamente modificate», in in Costato, Rook Basile e Germanò (diretto da), Trattato di diritto agrario. Volume secondo: il diritto agroambientale, Torino, p. 269324.

Teel J. (2000), Regulating genetically modified products and processes: an overview of approaches, in New York University of Environmental Law Journal, p. 649-703;

Valletta M. (2005), La disciplina delle biotecnologie agroalimentari, Milano 